\title{
PROJECTIONS ONTO TRANSLATION-INVARIANT SUBSPACES OF $L_{1}(R)$
}

BY

DALE E. ALSPACH ${ }^{1}$ AND ALEC MATHESON

ABSTRACT. The complemented translation-invariant subspaces of $L_{1}(\mathbf{R})$ are characterized. This completes an investigation begun by $\mathrm{H}$. P. Rosenthal.

Introduction. In the memoir [15] H. P. Rosenthal showed (among other things) that if a translation-invariant subspace $I$ of $L_{1}(\mathbf{R})$ is complemented, then the hull of $I, h I=\{t: \hat{f}(t)=0$ for all $f \in I\}$, is of the form

$$
\bigcup_{i=1}^{n}\left(\alpha_{i} \mathbf{Z}+\beta_{i}\right) \backslash F
$$

where $F$ is a finite set and $\left\{\alpha_{i}\right\}$ and $\left\{\beta_{i}\right\}$ are sequences of real numbers. In this paper we show that a necessary and sufficient condition for $I$ to be complemented is that $h I=\cup_{i=1}^{n}\left(\alpha_{i} \mathbf{Z}+\beta_{i}\right) \backslash F$ as above with $\left\{\alpha_{i}\right\}$ pairwise rationally dependent.

One consequence of this result is that each uncomplemented ideal with $h I=$ $\cup_{i=1}^{n}\left(\alpha_{i} \mathbf{Z}+\beta_{i}\right)$ is a $\mathcal{L}_{1, \lambda}$ subspace of $L_{1}(\mathbf{R})$ which is not isomorphic to $l_{1}$ or $L_{1}$. We do not know what the relationship is between these $\mathcal{L}_{1, \lambda}$ spaces and any of the previous constructions of such $\mathcal{L}_{1, \lambda}$ spaces $[\mathbf{1}, \mathbf{5}, \mathbf{8}]$.

This paper is divided into three parts. In the first we prove that the ideals with rationally dependent hull are complemented. In the second we complete the charaterization by showing that the existence of a projection onto an ideal with hull containing rationally independent cosets of $\mathbf{Z}$ would imply that $l_{1}$ and $l_{2}$ are isomorphic. In the third section we explore the isomorphic properties of the ideals as $\mathcal{E}_{1, \lambda}$ spaces.

We will use standard notation from Banach space theory as may be found in the book of Lindenstrauss and Tzafriri [11] and from harmonic analysis as found in the book of Katznelson [7]. The reader will also find the introduction and first section of Rosenthal's memoir [15] helpful.

Let us note that each of the sets $E=\cup_{i=1}^{n}\left(\alpha_{i} \mathbf{Z}+\beta_{i}\right) \backslash F$ is a set of spectral synthesis because it is closed and discrete. Thus $E$ uniquely determines an ideal $I(E)$ in $L_{1}(\mathbf{R})$, namely $I(E)=\left\{f:\left.\hat{f}\right|_{E}=0\right\}$. Spectral synthesis will play an important role in our argument involving the structure of $I(E)^{\perp}$. The sets $E$ of the above form coincide with the closed sets in the coset ring of $\mathbf{R}$ endowed with the discrete topology. These are exactly the strong Ditkin sets with no interior [16].

Received by the editors January 6, 1982 and, in revised form, June 22, 1982.

1980 Mathematics Subject Classification. Primary 46A15; Secondary 43A46, 46B20.

${ }^{1}$ Research supported in part by NSF grant MCS-802510.

(c) 1983 American Mathematical Society $0002-9947 / 82 / 0000-0686 / \$ 03.00$ 
We would like to thank H. P. Rosenthal for suggesting this problem and J. M. Rosenblatt for providing us with some unpublished results related to the problem.

1. The complemented ideals. In this section we will establish some general results about direct sum decompositions of $I(E)^{\perp}$ and show that $I(E)$ is complemented in the rationally dependent case. Many of the results in this section are known but a suitable reference does not seem to be available.

Definition. If $\alpha$ and $\beta$ are real numbers we will say that $\alpha$ and $\beta$ are rationally dependent if there exist integers $k$ and $l$ such that $k \alpha+l \beta=0$. We will denote this by $\alpha \equiv \beta$. If there are not such integers we will say that $\alpha$ and $\beta$ are rationally independent and denote this by $\alpha \neq \beta$.

LEMMA 1.1. If $E=\cup_{i=1}^{k}\left(\alpha_{i} \mathbf{Z}+\beta_{i}\right)$ where $\left\{\alpha_{i}\right\}$ and $\left\{\beta_{i}\right\}$ are sequences of real numbers, then $E=\cup_{j=1}^{m} E_{j}$, where for each $j$

$$
E_{j}=\bigcup_{k=1}^{n_{j}}\left(\theta_{j} \mathbf{Z}+\gamma_{j k}\right) \text {, }
$$

$\left\{\gamma_{j k}\right\}$ is a sequence of real numbers and the numbers $\theta_{1}, \theta_{2}, \ldots, \theta_{m}$ are pairwise rationally independent.

Proof. Let $\left\{\alpha_{i_{1}}, \alpha_{i_{2}}, \ldots, \alpha_{i_{m}}\right\}$ be a maximal set of pairwise rationally independent numbers in $\left\{\boldsymbol{\alpha}_{i}\right\}$. For each $j=1,2, \ldots, m$, let $E_{j}=\cup_{\boldsymbol{\alpha}_{i} \equiv \alpha_{i}}\left(\alpha_{i} \mathbf{Z}+\beta_{i}\right)$. For each fixed integer $j$ there exists a real number $\theta_{j}^{\prime}$ such that $\theta_{j}^{\prime} \equiv \alpha_{i j}$ and integers $k_{i}$ such that $k_{i} \theta_{j}^{\prime}=\alpha_{i}$ for each $i$ such that $\alpha_{i} \equiv \alpha_{i_{j}}$. Thus $E_{j}=\cup_{\alpha_{i} \equiv \alpha_{i}}\left(k_{i} \theta_{j}^{\prime} \mathbf{Z}+\beta_{i}\right)$.

Let $m_{j}$ be the least common multiple of the $k_{i}$ 's for $i$ such that $\alpha_{i} \equiv \alpha_{i_{j}}$, and let $\theta_{j}=m_{j} \theta_{j}^{\prime}$. Evidently

$$
k_{i} \theta_{j}^{\prime} \mathbf{Z}+\beta_{i}=\bigcup_{l=1}^{m_{j} k_{i}^{-1}}\left(\theta_{j} \mathbf{Z}+l k_{i} \theta_{j}^{\prime}+\beta_{i}\right)
$$

and so each $E_{j}$ has the required form.

REMARK. We may (and shall) assume that for fixed $j$, the cosets $\theta_{j} \mathbf{Z}+\gamma_{j k}$, $k=1,2, \ldots, n_{j}$, are disjoint.

LEMMA 1.2. If $\alpha$ and $\beta$ are real numbers, $\alpha \neq 0$, then $I(\alpha \mathbf{Z}+\beta)$ is complemented in $L_{1}(\mathbf{R})$ and

$$
I(\alpha \mathbf{Z}+\beta)^{\perp}=\left\{e^{i \beta x} g(x): g \in L_{\infty}(\mathbf{R}) \text { and } g \text { is periodic of period } 2 \pi \alpha^{-1}\right\} .
$$

Proof. Define $T: L_{1}(\mathbf{R}) \rightarrow L_{1}(\mathbf{R})$ by $T f(x)=\sum_{n \in \mathbf{Z}} f\left(x+2 \pi \alpha^{-1} n\right) 1_{\left[0,2 \pi \alpha^{-1}\right]}(x)$. The projection $P$ into $I(\alpha \mathbf{Z}+\beta)$ is then $P f(x)=f(x)-e^{i \beta x} T\left(e^{-i \beta s} f(s)\right)(x)$. Indeed, if $n$ is an integer,

$$
\begin{aligned}
\widehat{(I-P)} f(\alpha n+\beta) & =\int_{-\infty}^{\infty} e^{i \beta x} T\left(e^{-i \beta s} f(s)\right)(x) e^{-i(\alpha n+\beta) x} d x \\
& =\sum_{k \in \mathbf{Z}} \int_{0}^{2 \pi \alpha^{-1}} e^{i \beta\left(x+2 \pi \alpha^{-1} k\right)} f\left(x+2 \pi \alpha^{-1} k\right) e^{-i \alpha n x} d x \\
& =\sum_{k \in \mathbf{Z}} \int_{2 \pi \alpha^{-1} k}^{2 \pi \alpha^{-1}(k+1)} e^{-i \beta t} f(t) e^{-i \alpha n t} e^{i 2 \pi k n} d t \\
& =\hat{f}(\alpha n+\beta) .
\end{aligned}
$$


Consequently $\widehat{P f}(\alpha n+\beta)=0$ for all $n \in \mathbf{Z}$. Moreover it is easily seen that $I-P$ is a projection of $L_{1}(\mathbf{R})$ onto $L_{1}\left[0,2 \pi \alpha^{-1}\right]$, regarded as a subspace of $L_{1}(\mathbf{R})$. Since $\operatorname{supp}(I-P) f \subseteq\left[0,2 \pi \alpha^{-1}\right],(I-P) f=0$ if and only if $f \in I(\alpha n+\beta)$, by the uniqueness of the Fourier series. Hence $P$ is the required projection.

For the second assertion a simple application of the Hahn-Banach theorem proves that

$$
I(\alpha \mathbf{Z}+\beta)^{\perp}={\overline{\left[e^{i(\alpha n+\beta) x}: n \in \mathbf{Z}\right]}}^{w^{*}}=e^{i \beta x}{\overline{\left[e^{i \alpha n x}: n \in \mathbf{Z}\right]}}^{w^{*}} .
$$

Clearly a $w^{*}$ limit of $2 \pi \alpha^{-1}$-periodic functions is $2 \pi \alpha^{-1}$-periodic, proving the result.

REMARK. It follows from Lemma 2.2 and Corollary 1.3 of [15] that if $E=$ $\cup_{j=1}^{n}\left(\alpha \mathbf{Z}+\alpha q_{j}+\beta\right)$, where $\alpha, \beta \in \mathbf{R}$ and $\left\{q_{j}\right\} \subseteq Q$, then $I(E)$ is complemented. Indeed, there is a rational number $q$ such that $q^{-1} q_{j} \in \mathbf{Z}$ for $j=1,2, \ldots, n$, and thus $E \subset \alpha q \mathbf{Z}+\beta$. By identifying $\left[0,2 \pi(\alpha q)^{-1}\right]$ with $\mathbf{T}$, the circle group, we see that $E$ determines an ideal $\mathcal{G}$ in $L_{1}(\mathbf{T})$ with $h \mathcal{G}$ in the coset ring of $\mathbf{Z}$. Thus by [15, Corollary 1.3] and the remark following, 9 is complemented in $L_{1}(\mathbf{T})$. Finally $L_{1}(\mathbf{R})=I(E) \oplus$ $X$, where $X \subseteq L_{1}\left[0,2 \alpha(\pi q)^{-1}\right]$ is the complement of 9 .

Our next lemma is a consequence of the fact that $E=\cup_{i=1}^{k}\left(\alpha_{i} \mathbf{Z}+\beta_{i}\right)$ is a strong Ditkin set, however we will not use the terminology (see [9] for the definition). Applying the results of [9] to the set $E=\cup_{i=1}^{k}\left(\alpha_{i} \mathbf{Z}+\beta_{i}\right) \backslash F, F$ finite, we get the following

LEMmA 1.3. Let $E=\cup_{i=1}^{k}\left(\alpha_{i} \mathbf{Z}+\beta_{i}\right) \backslash F$. Then there is a sequence $\left\{\mu_{n}\right\}$ of measures on $\mathbf{R}$ such that:

(i) $\hat{\mu}_{n}=1$ on a neighborhood of $E$;

(ii) $\left\|\mu_{n} * f\right\| \rightarrow 0$ as $n \rightarrow \infty$ for all $f \in I(E)$;

(iii) $\left\|\mu_{n}\right\| \leqslant K<\infty$ for all $n$;

(iv) if $\rho \notin E$, then $\hat{\mu}_{n}(\rho)=0$ for $n$ sufficiently large.

LEMMA 1.4. Let

$$
E=\bigcup_{j=1}^{m} \bigcup_{k=1}^{n_{j}}\left(\left(\theta_{j} \mathbf{z}+\gamma_{j k}\right) \backslash F\right)
$$

where $\left(\boldsymbol{\theta}_{j} \mathbf{Z}+\gamma_{j k}\right) \cap\left(\boldsymbol{\theta}_{l} \mathbf{Z}+\gamma_{l s}\right) \subset F$ if $j \neq l$ or $k \neq s$. Then $I(E)^{\perp}$ is complemented in $L_{\infty}(\mathbf{R})$ and

$$
I(E)^{\perp}=\sum_{j=1}^{m} \sum_{k=1}^{n_{j}} \bigoplus I\left(\left(\theta_{j} \mathbf{Z}+\gamma_{j k}\right) \backslash F\right)^{\perp} .
$$

Proof. Fix $j$ and $k$. Let $\left\{\mu_{n}\right\}$ be the sequence of measures given by Lemma 1.3 for the set $\left(\theta_{j} \mathbf{Z}+\gamma_{j k}\right) \backslash F$, and define operators $P_{n}: L_{1}(\mathbf{R}) \rightarrow L_{1}(\mathbf{R})$ by $P_{n} f=\mu_{n} * f$. By (ii) of Lemma 1.3, $\left\|P_{n} f\right\| \rightarrow 0$ as $n \rightarrow \infty$ for all $f \in I\left(\left(\theta_{j} \mathbf{Z}+\gamma_{j k}\right) \backslash F\right)$. Let $P$ be a $w^{*}$ operator limit of $P_{n}^{*}$. Then $\langle P f, g\rangle=\lim _{n \rightarrow \infty}\left\langle f, P_{n} g\right\rangle=0$, if $g \in$ $I\left(\left(\theta_{j} \mathbf{Z}+\gamma_{j k}\right) \backslash F\right)$. Hence $P f \in I\left(\left(\theta_{j} \mathbf{Z}+\gamma_{j k}\right) \backslash F\right)^{\perp}$ for all $f \in L_{\infty}(\mathbf{R})$. If $f \in$ $\operatorname{span}\left\{e^{i\left(\theta_{j} n+\gamma_{j k}\right) x}: n \in \mathbf{Z}\right\}$, then $\langle P f, g\rangle=\lim _{n}\left\langle P_{n}^{*} f, g\right\rangle=\langle f, g\rangle$ for all $g \in L_{1}(\mathbf{R})$. Thus range $P=I\left(\left(\theta_{j} \mathbf{Z}+\gamma_{j k}\right) \backslash F\right)^{\perp}$ and $\left.P\right|_{\text {range } P}=\left.I\right|_{\text {Range } P}$. On the other hand, if 
$f \in \operatorname{span}\left\{e^{i \rho x}: \rho \in E \backslash\left(\theta_{j} \mathbf{Z}+\gamma_{j k}\right)\right\}$, then $\langle P f, g\rangle=\lim _{n}\left\langle f, P_{n} g\right\rangle=0$ for all $g \in$ $L_{1}(\mathbf{R})$, by (iv) of Lemma 1.3. Now if $f \in I\left(E \backslash\left(\theta_{j} Z+\gamma_{j k}\right)\right)^{\perp}$ and $K_{l}$ is a summability kernel with supp $\hat{K}_{l} \subseteq(-l, l)$, then

$$
\begin{aligned}
\langle P f, g\rangle & =\lim _{n}\left\langle f, P_{n} g\right\rangle=\lim _{n} \lim _{l}\left\langle f, P_{n}\left(K_{l} * g\right)\right\rangle \\
& =\lim _{l} \lim _{n}\left\langle f, P_{n}\left(K_{l} * g\right)\right\rangle=0 .
\end{aligned}
$$

It follows that

$$
\begin{aligned}
\operatorname{ker} P \cap I(E)^{\perp} & =\left\{f \in I(E)^{\perp}: \operatorname{supp} \hat{f} \backslash\left(\theta_{j} \mathbf{Z}+\gamma_{j k}\right)=\varnothing\right\} \\
& =\left\{f \in I(E)^{\perp}: \operatorname{supp} \hat{f} \subseteq\left(\theta_{j} \mathbf{Z}+\gamma_{j k}\right)\right\} \\
& =I\left(E \backslash\left(\theta_{j} \mathbf{Z}+\gamma_{j k}\right)\right)^{\perp},
\end{aligned}
$$

because these are sets of spectral synthesis. Clearly this implies that

$$
I(E)^{\perp}=\sum_{j=1}^{m} \sum_{k=1}^{n_{j}} \bigoplus I\left(\left(\theta_{j} \mathbf{Z}+\gamma_{j k}\right) \backslash F\right)^{\perp}
$$

and if we let $P_{j k}$ denote the projection constructed above for $\left(\theta_{j} \mathbf{Z}+\gamma_{j k}\right) \backslash F$, $k=1,2, \ldots, n$, then $\sum_{j=1}^{m} \sum_{k^{\prime}=1}^{n_{1}} P_{j k}$ is a projection onto $I(E)^{\perp}$.

(The fact that $I(E)^{\perp}$ is complemented was previously obtained by Gilbert [3].)

REMARK. The nature of the direct sum in the preceding lemma can be determined in some cases. For example, if $E=\cup_{j=1}^{m}\left(\theta_{j} \mathbf{Z} \backslash\{0\}\right)$ and $\left\{\boldsymbol{\theta}_{j}\right\}$ is independent over the rationals, then the direct sum is in fact equivalent to an $l_{1}$ sum (see [7, p. 185]). On the other hand, if $E=\cup_{j=1}^{m}(\mathbf{Z}+2 \pi j)$, then

$$
\sup \left\{\left\|\sum_{j=1}^{m} \varepsilon_{j} e^{i 2 \pi j t}\right\|_{\infty}:\left|\varepsilon_{j}\right|=1, j=1, \ldots, m\right\}=m,
$$

but $\inf \left\{\left\|\sum_{j=1}^{m} \varepsilon_{j} e^{i 2 \pi j t}\right\|_{\infty}:\left|\varepsilon_{j}\right|=1, \ldots, m\right\}=o(m)$. Indeed the Rudin-Shapiro polynomials [7, p. 33] yield $O(\sqrt{m})$, and recently Kahane [6] has given precise estimates. Y. Gordon and S. Reisner [4] have informed us of some related results.

Our next lemma is a simple consequence of standard results from Banach space theory.

Lemma 1.5. If $E \subseteq \mathbf{R}, E$ closed, and $F \subseteq \mathbf{R}, F$ finite, then $I(E)^{\perp}$ is $w^{*}$ complemented in $L_{\infty}(\mathbf{R})$ if and only if $I(E \cup F)^{\perp}$ is $w^{*}$ complemented in $L_{\infty}(\mathbf{R})$.

Our last lemma gives a method for building commuting projections in the rationally dependent case.

LEMMA 1.6. Let $E=\cup_{i=1}^{k}\left(\theta \mathbf{Z}+\beta_{i}\right)$, where $\left(\theta \mathbf{Z}+\beta_{i}\right) \cap\left(\theta \mathbf{Z}+\beta_{j}\right)=\varnothing$ for $i \neq j$. Then:

(a) For each $i$, there is a measure $\mu_{i} \in M(\mathbf{R})$ such that

$$
\hat{\mu}_{i}(y)= \begin{cases}1 & \text { if } y \in \theta \mathbf{Z}+\beta_{i}, \\ 0 & \text { if } y \in \theta \mathbf{Z}+\beta_{j}, j \neq i\end{cases}
$$


(b) for each $i$ there is a $w^{*}$ continuous projection $P_{i}^{*}$ of $L_{\infty}(\mathbf{R})$ onto $I\left(\theta \mathbf{Z}+\beta_{i}\right)$ such that $I\left(\theta \mathrm{Z}+\beta_{j}\right)^{\perp} \subseteq \operatorname{ker} P_{i}^{*}$ for $j \neq i$.

Proof. (a) Fix $i$. For each $j \neq i$ let

$$
v_{j}=\frac{1}{1-e^{2 \pi \theta^{-1} i\left(\beta_{i}-\beta_{j}\right)}}\left[\delta_{0}-e^{-2 \pi \theta^{-1} i \beta_{j}} \delta_{-2 \pi \theta^{-1}}\right] .
$$

Then

$$
\hat{v}_{j}(x)=\frac{1-e^{2 \pi \theta^{-1} i\left(x-\beta_{j}\right)}}{1-e^{2 \pi \theta^{-1} i\left(\beta_{i}-\beta_{j}\right)}}= \begin{cases}1 & \text { if } x \in \theta \mathbf{Z}+\beta_{i}, \\ 0 & \text { if } x \in \theta \mathbf{Z}+\beta_{j}, j \neq i .\end{cases}
$$

Let $\mu_{i}=v_{1} * v_{2} * \cdots * v_{i-1} * v_{i+1} * \cdots * v_{k}$. Clearly $\mu_{i}$ satisfies (a).

For (b), let $Q_{i}^{*}$ be the $w^{*}$ continuous projection of $L_{\infty}(\mathbf{R})$ onto $I\left(\theta \mathbf{Z}+\beta_{i}\right)^{\perp}$ given by Lemma 1.2 and define $P_{i}^{*} f=Q_{i}^{*}\left(\mu_{j} * f\right)$ for $f \in L_{\infty}(\mathbf{R})$. Just as in the proof of Lemma 1.4,

$$
\mu_{i} * f= \begin{cases}f & \text { if } f \in I\left(\theta \mathbf{Z}+\beta_{i}\right)^{\perp}, \\ 0 & \text { if } f \in I\left(\theta \mathbf{Z}+\beta_{j}\right)^{\perp}, \text { if } j \neq i .\end{cases}
$$

Hence

$$
P_{i}^{*}(f)= \begin{cases}f & \text { if } f \in I\left(\theta \mathbf{Z}+\beta_{i}\right)^{\perp} \\ 0 & \text { if } f \in I\left(\theta \mathbf{Z}+\beta_{j}\right)^{\perp}, \text { if } j \neq i\end{cases}
$$

as required.

We remark that part (b) is essentially contained in Theorem 5 of [12].

We are now ready to prove the main result of this section.

THEOREM A. Suppose $E=\cup_{i=1}^{k}\left(\theta_{i} \mathbf{Z}+\gamma_{i}\right) \backslash F$, where $F$ is a finite set, $\left\{\boldsymbol{\theta}_{i}\right\}$ and $\left\{\gamma_{i}\right\}$ are sequences of real numbers and $\theta_{i} \equiv \theta_{j}$ for all $i$ and $j$. Then $I(E)$ is a complemented subspace of $L_{1}(\mathbf{R})$.

Proof. First note that $I(E)$ is complemented in $L_{1}(\mathbf{R})$ if and only if $I(E)^{\perp}$ is $w^{*}$ complemented in $L_{\infty}(\mathbf{R})$. By Lemma 1.5 we may assume that $F=\varnothing$ and by Lemma 1.1 that $E=\cup_{i=1}^{n}\left(\theta \mathbf{Z}+\beta_{i}\right)$, where $\left(\theta \mathbf{Z}+\beta_{i}\right) \cap\left(\theta \mathbf{Z}+\beta_{j}\right)=\varnothing$ if $i \neq j$. Finally by Lemma $1.6(\mathrm{~b})$ there is a sequence of $w^{*}$ continuous projections $\left\{P_{i}\right\}$ such that for each $i, P_{i}$ projects $L_{\infty}(\mathbf{R})$ onto $I\left(\theta \mathbf{Z}+\beta_{i}\right)^{\perp}$ and ker $P_{i} \supseteq I\left(\theta \mathbf{Z}+\beta_{j}\right)^{\perp}$ for $j \neq i$. Hence $P=\sum_{i=1}^{n} P_{i}$ is a $w^{*}$ continuous projection of $L_{\infty}(\mathbf{R})$ onto $\sum_{i=1}^{n} I\left(\theta \mathbf{Z}+\beta_{i}\right)^{\perp}=$ $I\left(\cup_{i=1}^{n}\left(\theta \mathbf{Z}+\beta_{i}\right)\right)^{\perp}$.

2. Uncomplemented ideals. In [15] Rosenthal shows that if $E \subseteq \mathbf{R}$ is closed, then a necessary condition for an ideal $I$ with $h I=E$ to be complemented in $L_{1}(\mathbf{R})$ is that $E$ belong to the coset ring of $\mathbf{R}$ with the discrete topology. He then shows that such an $E$ has the form $\cup_{i=1}^{k}\left(\alpha_{i} Z+\beta_{i}\right) \backslash F$, where $\left\{\alpha_{i}\right\}$ and $\left\{\beta_{i}\right\}$ are sequences of real numbers, and $F$ is a finite set. In the previous section we proved that $I(E)$ is complemented if the $\alpha_{i}$ 's are pairwise rationally dependent. In this section we will show that this is a necessary condition. 
The proof will be by contradiction, that is, we will assume that there is a projection $Q$ of $L_{1}(\mathbf{R})$ onto $I(E)$, where $E=\cup_{i=1}^{k}\left(\alpha_{i} \mathbf{Z}+\beta_{i}\right)$ and (say) $\alpha_{1} Z \alpha_{2}$. (Lemma 1.5 allows us to ignore the finite set $F$.) We will then show that the fact that the cosets $\alpha_{1} \mathbf{Z}+\beta_{1}$ and $\alpha_{2} \mathbf{Z}+\beta_{2}$ have points arbitrarily close together induces an isomorphism from $l_{1}$ to $l_{2}$.

Lemma 2.1. Let $A_{1}, A_{2}, \ldots, A_{n}$ be subspaces of $L_{1}(\mathbf{R})$ such that $X=\left[A_{i}^{\perp}: i=\right.$ $1,2, \ldots, n]$ is $w^{*}$ complemented in $L_{\infty}(\mathbf{R})$ and $X=\sum_{i=1}^{n} \oplus A_{i}^{\perp}$. Then there is a projection $P$ of $L_{1}(\mathbf{R})$ onto $A_{1}$ such that $\operatorname{ker} P \subseteq \cap_{i=2}^{n} A_{i}$.

Proof. Each of the subspaces $A_{\mathrm{i}}^{\perp}$ of $L_{\infty}(\mathbf{R})$ is $w^{*}$ closed and thus $X$ is a $w^{*}$ direct sum of the $A_{i}^{\perp}$ 's. Therefore there is a $w^{*}$ continuous projection $R^{*}$ of $X$ onto $A_{1}^{\perp}$ such that ker $R^{*} \supseteq \cup_{i=2}^{n} A_{i}^{\perp}$. Let $Q^{*}$ be the $w^{*}$ continuous projection of $L_{\infty}(\mathbf{R})$ onto $X$ and let $P^{*}=I-R^{*} Q^{*}$.

If $f \in A_{1}$ and $g \in L_{\infty}(\mathbf{R})$, then

$$
\langle g, P f\rangle=\left\langle\left(I-R^{*} Q^{*}\right) g, f\right\rangle=\langle g, f\rangle-\left\langle R^{*} Q^{*} g, f\right\rangle=\langle g, f\rangle .
$$

Hence $\left.P\right|_{A_{1}}=\left.I\right|_{A_{1}}$ and clearly $P\left(L_{1}(\mathbf{R})\right) \subseteq A_{1}$.

If $h \in \operatorname{ker} P$ and $g \in L_{\infty}(\mathbf{R})$, then $0=\langle g, P h\rangle=\left\langle\left(I-R^{*} Q^{*}\right) g, h\right\rangle$. If now $g \in$ $A_{i}^{\perp}$, for some $i=2,3, \ldots, n$, then $R^{*} Q^{*} g=R^{*} g=0$. Thus $\langle g, h\rangle=0$, i.e., $h \in$ $\bigcap_{i=2}^{n}\left(A_{i}^{\perp}\right)_{\perp}=\bigcap_{i=2}^{n} A_{i}$.

For the remainder of this section, $S=S(\alpha)$ will denote the operator from $L_{1}(\mathbf{R})$ onto $L_{1}\left[0,2 \pi \alpha^{-1}\right]$ defined by

$$
S f(x)=\sum_{n \in \mathbf{Z}} f\left(x+2 \pi \alpha^{-1} n\right) 1_{\left[0,2 \pi \alpha^{-1}\right]}(x) .
$$

Note that $S$ is a projection onto $L_{1}\left[0,2 \pi \alpha^{-1}\right], \widehat{S f}(\alpha n)=\hat{f}(\alpha n)$ for all $n \in \mathbf{Z}$ and $f \in L_{1}(\mathbf{R})$, and $\operatorname{ker} S=I(\alpha \mathbf{Z})$. (See the proof of Lemma 1.2.)

LEMMA 2.2. If $P$ is a projection from $L_{1}(\mathbf{R})$ onto $I(\alpha \mathbf{Z})$, then $\left.S\right|_{k e r}$ is an isomorphism onto $L_{1}\left[0,2 \pi \alpha^{-1}\right]$.

Proof. The projection $S$ defines a decomposition $L_{1}(\mathbf{R})=I(\mathbf{Z}) \oplus L_{1}[0,2 \pi]$ and the projection $P$ defines another decomposition $L_{1}(\mathbf{R})=I(\mathbf{Z}) \oplus X$ where $X=\operatorname{ker} P$. Because $\operatorname{ker} S=I(\mathbf{Z}),\left.S\right|_{X}$ is one-to-one. The range of $\left.S\right|_{X}$ equals $S\left(L_{1}(\mathbf{R})\right)=$ $L_{1}[0,2 \pi]$. Hence $\left.S\right|_{X}$ is an isomorphism onto $L_{1}[0,2 \pi]$ as claimed.

The next lemma is really the heart of the proof that $I(E)$ is not complemented when $E$ contains rationally independent cosets.

LEMMA 2.3. There is no projection $P$ of $L_{1}(\mathbf{R})$ onto $I(\alpha \mathbf{Z})$ such that ker $P \subseteq$ $I\left(\left(\alpha^{\prime} \mathbf{Z}+\beta\right) \backslash F\right)$ where $\alpha \neq \alpha^{\prime}$ and $F$ is a finite set.

Proof. Suppose $P$ is such a projection. Then by Lemma 2.2, if $X=\operatorname{ker} P,\left.S\right|_{X}$ is an isomorphism onto $L_{1}\left[0,2 \pi \alpha^{-1}\right]$. Let $x_{n}=\left(\left.S\right|_{X}\right)^{-1} e^{i \alpha n x} 1_{\left[0,2 \pi \alpha^{-1}\right]}(x), n=1,2, \ldots$

Claim. For every $k \in \mathbf{N}$ there is an $n \in \mathbf{N}$ such that $\left\|x_{n}-x_{n} 1_{[-k, k]}\right\| \geqslant \pi \alpha^{-1} / 2$.

First observe that if $t_{1}, t_{2} \in \mathbf{R}$, then

$$
\left\|\widehat{x_{n} 1_{[-k, k]}}\left(t_{1}\right)-\widehat{x_{n} 1_{[-k, k]}}\left(t_{2}\right)|\leqslant| k|| t_{1}-t_{2} \mid\right\|\left(\left.S\right|_{X}\right)^{-1} \| 2 \pi \alpha^{-1},
$$


because

$$
\left|\frac{d}{d t} \widehat{x_{n} 1_{[-k, k]}}(t)\right| \leqslant|k|\left\|x_{n}\right\| .
$$

Because $\alpha \not \alpha^{\prime}$, for every $\delta>0$ there are integers $n_{1}$ and $n_{2}$ such that $\mid \alpha n_{1}-\left(\alpha^{\prime} n_{2}\right.$ $+\beta) \mid<\delta$ and $\alpha^{\prime} n_{2}+\beta \notin F$. We have that $\hat{x}_{n_{1}}\left(a n_{1}\right)=\widehat{S x}_{n_{1}}\left(\alpha n_{1}\right)=2 \pi \alpha^{-1}$ and $\hat{x}_{n_{1}}\left(\alpha^{\prime} n_{2}+\beta\right)=0$. Thus if $\delta$ is sufficiently small, either

$$
\left|\hat{x}_{n_{1}}\left(\alpha n_{1}\right)-\widehat{x_{n_{1}} 1_{[-k, k]}}\left(\alpha n_{1}\right)\right| \geqslant \frac{1}{4}\left(2 \pi \alpha^{-1}\right) \text {, }
$$

or

$$
\left|\hat{x}_{n_{1}}\left(\alpha^{\prime} n_{2}+\beta\right)-x_{n_{1}} 1_{[-k, k]}\left(\alpha^{\prime} n_{2}+\beta\right)\right| \geqslant \frac{1}{4}\left(2 \pi \alpha^{-1}\right) .
$$

In either case $\left\|x_{n_{1}}-x_{n_{1}} 1_{[-k, k]}\right\|>\pi \alpha^{-1} / 2=\rho$, proving the claim.

It follows by a standard gliding hump argument that there is a subsequence $\left\{x_{n_{l}}\right\}$ of $\left\{x_{n}\right\}$ and a strictly increasing sequence of integers $\left\{k_{l}\right\}$ such that

(i) $\left\|x_{n_{l}} 1_{\left[-k_{l},-k_{l-1}\right] \cup\left[k_{l-l}, k_{l}\right]}\right\|>\rho / 2$, and

(ii) $n_{l}>3 n_{l-1}$

for each $l \in \mathbf{N}$. By Rosenthal's disjointness lemma [13], there is a further subsequence of $\left\{x_{n_{l}}\right\},\left\{x_{n_{l}}\right\}_{l \in M}, M$ is infinite, which is equivalent to the usual unit vector basis of $l_{1}$. On the other hand $\left\{S x_{n_{l}}\right\}_{l \in M}$ is a lacunary sequence of exponentials $\left\{e^{i \alpha n_{l} x} 1_{\left[0,2 \pi \alpha^{-1}\right]}\right\}_{l \in M}$ and thus is equivalent to the usual unit vector basis of $l_{2}$. This contradicts the assertion that $S$ is an isomorphism on $X$.

We are now ready to prove

THEOREM B. Let $E=\cup_{i=1}^{k}\left(\alpha_{i} \mathbf{Z}+\beta\right) \backslash F$ where $\left\{\alpha_{i}\right\}$ and $\left\{\beta_{i}\right\}$ are sequences of real numbers, $F$ is a finite set, and the $\alpha_{i}$ are not pairwise rationally dependent. Then $I(E)$ is not complemented in $L_{1}(\mathbf{R})$.

PRoof. By Lemmas 1.1 and 1.5 we may assume that $\left(\alpha_{i} \mathbf{Z}+\beta_{i}\right) \cap\left(\alpha_{j} \mathbf{Z}+\beta_{j}\right) \subseteq F$ where $i \neq j$. Hence by Lemma 1.4

$$
I(E)^{\perp}=\sum_{i=1}^{k} \bigoplus I\left(\left(\alpha_{i} \mathbf{Z}+\beta_{i}\right) \backslash F\right)^{\perp} .
$$

Clearly we may also assume that $\alpha_{1} \neq \alpha_{2}$ and that $\beta_{1}=0$.

If $I(E)$ is complemented in $L_{1}(\mathbf{R})$, it follows that $I(E)^{\perp}$ is $w^{*}$ complemented in $L_{\infty}(\mathbf{R})$, and so, by Lemma 2.1 , there is a projection $P$ of $L_{1}(\mathbf{R})$ onto $I\left(\alpha_{1} Z \backslash F\right)$ with ker $P \subseteq I\left(\left(\alpha_{2} Z+\beta_{2}\right) \backslash F\right)$. Because $F$ is finite, $I\left(\alpha_{1} Z \backslash F\right) / I\left(\alpha_{1} Z\right)$ is finite dimensional. (Since $I\left(\alpha_{1} Z \backslash F\right) \cap I\left(\left(\alpha_{2} Z+\beta_{2}\right) \backslash F\right)$ contains a copy of $L_{1}$, and $I\left(\alpha_{1} Z \backslash F\right)$ $\cap I\left(\left(\alpha_{2} \mathbf{Z}+\beta_{2}\right) \backslash F\right) \cap \operatorname{ker} P=\{0\}$, it follows that $I\left(\alpha_{2} \mathbf{Z} \backslash F\right) / \operatorname{ker} P$ is finite dimensional. See Proposition 3.1.) Hence we may assume that $P$ is a projection onto $I\left(\alpha_{1} \mathbf{Z}\right)$ such that $\operatorname{ker} P \subseteq I\left(\left(\alpha_{2} \mathbf{Z}+\beta_{2}\right) \backslash F\right)$. But this contradicts Lemma 2.3 with $\alpha=\alpha_{1}, \alpha^{\prime}=\alpha_{2}$, and the theorem follows.

3. The ideals as $\mathcal{L}_{1, \lambda}$ spaces. In this section we will show that if

$$
E=\bigcup_{i=1}^{k}\left(\alpha_{i} \mathbf{Z}+\beta_{i}\right) \backslash F,
$$


then $I(E)$ is a $\varrho_{1, \lambda}$ space and contains a complemented isomorphic copy of $L_{1}$. These are not new results, but combining them with the results of the previous section gives a possibly new set of $\varrho_{1, \lambda}$ spaces.

Proposition 3.1. Let $E=\cup_{i=1}^{k}\left(\alpha_{i} \mathbf{Z}+\beta_{i}\right) \backslash F$ where $\left\{\alpha_{i}\right\}$ and $\left\{\beta_{i}\right\}$ are sequences of real numbers, $F$ is a finite set, and $\left\{\alpha_{i}\right\}$ are not pairwise rationally dependent. Then

(i) $L_{1}$ is isomorphic to a complemented subspace of $I(E)$,

(ii) $I(E)$ is a $L_{1, \lambda}$ space,

(iii) $I(E)$ is not isomorphic to $L_{1}$.

Proof. We may assume that $\alpha_{i}>0, i=1,2, \ldots, k$. For each $i \leqslant k$, let $\mu_{i}=\delta_{0}-$ $e^{-\alpha_{i}^{-1} 2 \pi \beta_{i}} \delta_{-2 \pi \alpha^{-1}}$. Define an operator $T$ on $L_{1}(\mathbf{R})$ by $T f=\mu_{1} * \mu_{2} * \cdots * \mu_{k} * f$. If $0<s<\min 2 \pi \alpha_{i}^{-1}$, then $\left.T\right|_{L_{1}[0, s]}$ is an isomorphism and $T L_{1}(\mathbf{R}) \subset I(E)$. Indeed $\mu_{i} * g(x)=g(x)-e^{-i \alpha_{i}^{-1} 2 \pi \beta_{i}} g\left(x+2 \pi \alpha_{i}^{-1}\right)$, and thus if supp $f \subseteq[0, s],\left.\left(\mu_{i} * f\right)\right|_{[0, s]}$ $=f$, while if $\operatorname{supp} f \subseteq(s, \infty),\left.\left(\mu_{i} * f\right)\right|_{[0, s]}=0$. Hence $\|T f\| \geqslant\|f\|$ for $f \in L_{1}[0, s]$. To see that $T L_{1}(\mathbf{R}) \subseteq I(E)$ it suffices to note that

$$
\widehat{T f}(x)=\prod_{i=1}^{k}\left(1-e^{i \alpha_{i}^{-1} 2 \pi\left(x-\beta_{i}\right)}\right) \hat{f}(x) .
$$

Moreover $T L_{1}[0, s]$ is complemented by the projection $P f=T\left(\left.f\right|_{[0, s]}\right)$.

To see (ii), it follows from Lemma 1.4 that $I(E)^{\perp}$ is complemented in $L_{\infty}(\mathbf{R})$ and so $I(E)^{*}=L_{\infty}(R) / I(E)^{\perp}$ is isomorphic to the complement of $I(E)^{\perp}$. It is well known that every complemented subspace of $L_{\infty}(\mathbf{R})$ is isomorphic to $L_{\infty}(\mathbf{R})[\mathbf{1 1}, \mathrm{p}$. 57]. Thus $I(E)^{*}$ is a $\mathscr{L}_{\infty, \lambda}$ space and hence $I(E)$ is a $\mathscr{L}_{1, \lambda}$ space [10].

Finally by the results of $\S 2, I(E)$ is not complemented. Because $L_{1}(\mathbf{R}) / I(E)$ is a $\varrho_{1, \lambda}$ space, a result of Lindenstrauss [9] implies that $I(E)$ is not complemented in a second conjugate space. Thus $I(E)$ is not isomorphic to $L_{1}$.

All of the previous constructions $[\mathbf{1 , 5}, \mathbf{8}]$ of $\mathcal{L}_{1, \lambda}$ spaces gave examples which do not contain $L_{1}$ and so are not isomorphic to the ideals above. However it is possible that the ideals are simply direct sums of $L_{1}$ with the previously known examples.

We ask the following questions:

1. If $\alpha_{1} Z \alpha_{2}$, is $I\left(\alpha_{1} \mathbf{Z} \cup \alpha_{2} Z\right)$ isomorphic to $I(Z \cup \sqrt{ } 2 Z)$ ?

2. More generally, what is the isomorphic classification of the ideals

$$
I\left(\bigcup_{i=1}^{k}\left(\boldsymbol{\alpha}_{i} \mathbf{z}+\beta_{i}\right)\right) ?
$$

The authors with J. M. Rosenblatt have obtained some extensions of these results to $L_{1}(G)$ where $G$ is a locally compact abelian group. These results will appear in a forthcoming paper.

\section{REFERENCES}

1. J. Bourgain, $A$ new class of $\mathrm{L}^{1}$-spaces, Israel J. Math 39 (1981), 113-125.

2. J. E. Gilbert, On a strong form of spectral synthesis, Ark. Mat. (7) 43 (1968), 571-575. 3. $\ldots$, On projections of $L^{\infty}(G)$ onto translation-invariant subspaces, Proc. London Math. Soc. (3) 19 (1969), 69-88.

4. Y. Gordon and S. Reisner, Some geometrical properties of Banach spaces of polynomials, preprint. 5. W. B. Johnson and J. Lindenstrauss, Examples of $\mathcal{L}_{1}$ spaces, preprint. 
6. J. P. Kahane, Sur les polynômes à coefficients unimodulaires, Bull. London Math. Soc. 12 (1980), $321-342$.

7. Y. Katznelson, An introduction to harmonic analysis, Dover, New York, 1976.

8. J. Lindenstrauss, $A$ remark on $\mathcal{L}_{1}$ spaces, Israel J. Math. 8 (1970), 80-82.

9. __ On certain subspaces of $l_{2}$, Bull. Acad. Polon. Sci. Sér. Sci. Math. 12 (1964), 539-542.

10. J. Lindenstrauss and H. P. Rosenthal, The $\varrho_{p}$ spaces, Israel J. Math. 7 (1969), 325-349.

11. J. Lindenstrauss and L. Tzafriri, Classical Banach spaces. I, Springer-Verlag, Berlin and New York, 1977.

12. Teng-Sun Liu, Arnould Van Rooij and Ju-Kwei Wang, Projections and approximate identities for ideals in group algebras, Trans. Amer. Math Soc. 175 (1973), 469-482.

13. H. P. Rosenthal, On relatively disjoint families of measures, with some applications to Banach space theory, Studia Math. 37 (1970), 13-36.

14. $\ldots$, On the existence of approximate identities in ideals of group algebras, Ark. Mat. 7 (1967), $185-191$.

15. _ Projections onto translation-invariant subspaces of $L^{p}(G)$, Mem. Amer. Math. Soc. No. 63, (1966).

16. S. Saeki, On strong Ditkin sets, Ark. Mat. 10 (1972), 1-7.

17. Bert M. Schreiber, On coset rings and strong Ditkin sets, Pacific J. Math. 3 (1970), 805-812.

Department of Mathematics, Oklahoma State University, Stillwater, OKlahoma 74078 